\title{
BMJ Open Changes in cardiovascular health score and atherosclerosis progression in middle-aged and older persons in China: a cohort study
}

Jingsheng Gao, ${ }^{1}$ Minghui Bao, ${ }^{2}$ Yan Liu, ${ }^{1}$ Jihong Shi, ${ }^{1}$ Zhe Huang, ${ }^{1}$ Aijun Xing, ${ }^{1}$ Yang Wang, ${ }^{1}$ Shasha An, ${ }^{1}$ Jun Cai, ${ }^{2}$ Shouling Wu, ${ }^{1}$ Xinchun Yang ${ }^{2}$

To cite: Gao J, Bao M, Liu Y, et al. Changes in cardiovascular health score and atherosclerosis progression in middle-aged and older persons in China: a cohort study. BMJ Open 2015;5:e007547. doi:10.1136/bmjopen-2014007547

- Prepublication history and additional material is available. To view these files please visit the journal (http://dx.doi.org/10.1136/ bmjopen-2014-007547).

SW and $X Y$ contributed equally.

$J G$ and $M B$ contributed equally.

Received 26 December 2014 Revised 15 April 2015 Accepted 20 April 2015

\section{CrossMark}

\footnotetext{
${ }^{1}$ Department of Cardiology, Kailuan Hospital, Hebei United University, Tangshan, China

${ }^{2}$ Department of Cardiology, Chaoyang Hospital, Capital Medical University, Beijing, China
}

Correspondence to Professor Xinchun Yang; yxc6229@sina.com

\section{ABSTRACT}

Objectives: The American Heart Association (AHA) proposed a definition of 4 cardiovascular health behaviours and 3 health factors. On the basis of the 7 metrics, the cardiovascular health score (CHS) was used to estimate individual-level changes in cardiovascular health status. The aim of this study was to investigate whether changes in $\mathrm{CHS}(\triangle \mathrm{CHS})$ at different time-points are associated with atherosclerosis progression in middle-aged and older persons.

Design: Prospective cohort study in China.

Settings: We defined 8 groups $(\leq-4,-3,-2,-1,0$, 1,2 and $\geq 3$ ) according to $\triangle \mathrm{CHS}$. The impact of $\triangle \mathrm{CHS}$ on the change of brachial-ankle pulse wave velocity ( $\triangle \mathrm{baPWV}$ ) and atherosclerosis progression was analysed.

Participants: A total of 3951 individuals met the inclusion criteria ( $\geq 40$ years old; no history of stroke, transient ischaemic attack or myocardial infarction) and had complete information.

Results: $\triangle$ baPWV decreased gradually (126.46 $\pm 355.91,78.4 \pm 343.81,69.6 \pm 316.27,49.59 \pm 287.57$, $57.07 \pm 261.17,40.45 \pm 264.27,37.45 \pm 283.26$ and 21.66 $\pm 264.17 \mathrm{~cm} / \mathrm{s}$, respectively) with increasing $\triangle \mathrm{CHS}$ ( $p$ for trend $<0.05$ ). Multivariate linear regression analysis suggested a negative relationship between these 2 variables, which persisted after adjustment for other risk factors. Each increase in $\mathrm{CHS}$ was associated with a reduced baPWV for $15.22 \mathrm{~cm} / \mathrm{s}$ (B value -15.22 , $p<0.001$ ).

Conclusions: $\triangle \mathrm{CHS}$ were negatively related to $\triangle$ baPWV, which proved to be an independent predictor of the progression of atherosclerosis in middle-aged and older persons.

Trial registration number: Kailuan study (ChiCTRTNC-11001489).

\section{INTRODUCTION}

Cardiovascular disease (CVD) is the leading cause of morbidity and mortality worldwide. ${ }^{12}$ Several risk factors, such as cigarette smoking, excessive alcohol consumption, imbalanced diet, obesity and physical inactivity, can promote the development of CVD and

\section{Strengths and limitations of this study}

- Measurements taken twice to estimate individuallevel changes in cardiovascular health status.

- The present study explored the changes in cardiovascular health status, as well as investigated atherosclerosis progression which was evaluated by brachial-ankle pulse wave velocity.

- We did not adhere perfectly to all of the American Heart Association (AHA) 2020 health metrics for practical reasons.

- The duration of follow-up was 2 years, which was not long enough to fully track the progression of atherosclerosis.

- The results of the study are based on middle-aged and older persons, but whether it can be generalised to the whole population warrants further validation.

premature mortality. ${ }^{3}$ Avoiding these risk factors and adopting healthy lifestyle changes might result in a lower CVD incidence. ${ }^{4}$

In 2010, the American Heart Association (AHA) proposed its first definition of cardiovascular health behaviours and health factors. $^{2}$ This definition consists of seven metrics-four health behaviours (smoking, diet, physical activity and body mass index (BMI)) and three health factors (plasma glucose, cholesterol and blood pressure (BP) - - that are used to categorise individuals into 'poor', 'intermediate' and 'ideal' groups. To estimate individual-level changes in cardiovascular health behaviours and health factors, Huffman $^{5}$ established the AHA cardiovascular health score (CHS), which includes all seven cardiovascular health behaviours and health factors (each factor scored as-poor, 0 point; intermediate, 1 point; or ideal, 2 points -total scale: 0-14 points). Several studies have since detected a protective effect of ideal cardiovascular health behaviours and 
health factors on the incidence of cardiovascular and cerebrovascular diseases. The risk of cardiovascular and cerebrovascular diseases, all-cause mortality and cardiovascular mortality dramatically declines with increasing values for cardiovascular health metrics. ${ }^{36}$ Moreover, cardiovascular health status conversion from 'poor' or 'intermediate' to 'ideal' might also benefit the cardiovascular and cerebrovascular systems by reducing the odds of detectable coronary artery calcification and lowering the intima-media thickness. ${ }^{7}$ Unfortunately, both the prevalence of ideal cardiovascular health status and the improvement of CHS are far from satisfactory. ${ }^{28}$

Atherosclerosis is a chief factor contributing to the occurrence and development of CVD. Arterial stiffness is a predictor of atherosclerosis and CVD, ${ }^{9}$ which can be evaluated by pulse wave velocity (PWV). Among multiple methods used to measure $\mathrm{PWV},{ }^{10}$ brachial-ankle PWV (baPWV) can be measured much more conveniently and non-invasively ${ }^{11}$ and has the potential to be widely used in large-scale clinical studies. Positive relationships between baPWV and CVD have been detected in several studies. ${ }^{12-15}$

Taking into consideration these findings, the protective effect of CHS on the incidence of CVD might be mediated by its favourable effects on baPWV. To the best of our knowledge, however, the relationship between CHS changes and baPWV changes has not been reported. Therefore, on the basis of the population of the Chinese Kailuan study (ChiCTR-TNC-11001489), we adopted baPWV as an index to investigate whether changes in CHS ( $\Delta$ CHS) can affect the progression of atherosclerosis in middle-aged and older persons.

\section{MATERIALS AND METHODS \\ Study participants}

Eleven hospitals in China participated in the physical examination. A total of four physical examinations were performed during 2006-2007, 2008-2009, 2010-2011 and 2012-2013, respectively. The measurement of baPWV was added into the last two examinations. The examination was performed on both in-service and retired workers between June 2006 and October 2007. A total of 101510 workers participated in the physical examination (81 110 men and 20400 women).

\section{Inclusion and exclusion criteria}

Participants were included if they were $\geq 40$ years old and provided informed consent for the present study. Participants were excluded if they had history of stroke (except for lacunars infarction), had history of transient ischaemic attack, had history of myocardial infarction, had incomplete information (cardiovascular health metrics, baPWV), had extreme values of baPWV at the third or the fourth examination or died during follow-up.
Data collection

\section{Epidemiological questionnaire}

The questionnaire was completed by trained researchers or doctors on the day of the health examination. The questionnaire items consisted of demographic information, occupation situation, lifestyle (eg, cigarette smoking, exercise and diet), disease history and family history, and physical examination profiles (eg, BP, height, weight, waist circumference, etc.). Smoking was defined as $\geq 1$ cigarette/day, continuous smoking $\geq 1$ year, or giving up smoking $\leq 1$ year.

\section{Anthropometric and biochemical measurements}

Standard protocols were used for all the measurements as described earlier by our group. ${ }^{16}$ Anthropometric measurements included the measurements of height, weight, waist circumference, hip circumference, BMI, and BP. Biochemical measurements: fasting blood glucose (FBG), triglyceride (TG), total cholesterol (TC), high-density lipoprotein cholesterol (HDL-C), low density lipoprotein cholesterol (LDL-C), etc, (see online supplementary method 1 for details).

\section{baPWV measurement}

Measurements were repeated twice for each people and the second data was regarded as the final record. We analysed the larger value of the left and right side. The changes of baPWV ( $\triangle \mathrm{baPWV})$ were calculated as the baPWV value in examination of 2012-2013 minus the value in 2010-2011. Individuals were divided into 2 groups according to $\triangle \mathrm{baPWV}$. Participants with $\triangle \mathrm{baPWV} \leq 0$ were defined as non-increasing group, and participants with $\triangle \mathrm{baPWV}>0$ were defined as increasing group (see online supplementary method 2 for details).

\section{Cardiovascular health metrics}

According to the cardiovascular health behaviours and health factors proposed by $\mathrm{AHA}^{2}{ }^{2}$ and the scoring system (the AHA CHS) raised by Huffman, ${ }^{5}$ the seven cardiovascular health behaviours and health factors were divided into three levels (poor $=0$ point; intermediate $=1$ point; and ideal=2 points-total scale: $0-14$ points). Owing to lacking of detailed diet data in the original scale, and taken into account the great influence of salt intake on CVD among Chinese population, we adopted salt intake as a surrogate of diet. The definition of exercise is slightly different with which proposed by AHA (the ideal amount of exercise proposed by AHA is more than 5 times per week and more than $30 \mathrm{~min}$ each time) (see online supplementary method 3 for details).

\section{Grouping method}

We adopted the scoring system named CHS proposed by Huffman. Assignment of cardiovascular health metrics: poor, 0 point; intermediate, 1 point; ideal, 2 pointstotal scale: $0-14$ points. The $\triangle \mathrm{CHS}$ : the CHS in examination of 2012-2013 minus the CHS in 2010-2011. We divided population into eight groups $(\leq-4,-3,-2,-1$, 
$0,1,2$ and $\geq 3$ ) and classified them into three groups: decreasing group $(\triangle \mathrm{CHS}<0)$, invariant group $(\triangle \mathrm{CHS}=0)$, and increasing group $(\Delta \mathrm{CHS}>0)$.

\section{Data management and statistical method}

EpiData software was used to establish the data base. SPSS 13.0 statistical software was used for statistical analysis. Normally distributed data was recorded as (mean $\pm \mathrm{SD}$ ). Variance analysis was used when more than two groups were compared. The mean was compared by the method of Least Significant Difference (LSD) (homogeneous variance) or Welch (heterogeneous variance). Categorical variables were described as percentages and compared using $\chi^{2}$. Multivariate linear regression analysis was used to investigate the relationship between $\triangle \mathrm{CHS}$ and $\triangle \mathrm{baPWV}$. The $\mathrm{p}<0.05$ (bilateral) was regarded as statistically significant.

\section{Missing values replacement}

We adopted data of the first, second and third physical examination as replacement of the missing values of the third and fourth examination. The extreme value of baPWV refers to values beyond the 99 centile. The extreme value of the third examination is $2829 \mathrm{~cm} / \mathrm{s}$ (the left side) and $2707 \mathrm{~cm} / \mathrm{s}$ (the right side). The extreme value of the fourth examination is $3734 \mathrm{~cm} / \mathrm{s}$ (the left side) and $3955 \mathrm{~cm} / \mathrm{s}$ (the right side) (see online supplementary method 4 for details). In order to reduce the bias and improve the authenticity of our results, we excluded the extreme values of baPWV for the consideration that these extreme values might be due to the measurement errors.

\section{RESULTS}

From 2010 to 2011, a sample of 5852 participants older than 40 years was randomly selected from 101510 workers. In total, 5816 participants eventually completed the third physical examination. Among them, 376 participants did not meet the following inclusion criteria: no history of stroke, transient ischaemic attack or myocardial infarction. Of the remaining 5440 individuals, 445 did not participate in the fourth examination. About 243 had incomplete information (baPWV, cardiovascular health metrics) and extreme value of baPWV at the third examination, and 786 at the fourth examination. Fifteen individuals died during the follow-up. Finally, 3951 participants were included in the statistical analysis (2267 men and 1684 women). See figure 1 for detailed information of participants inclusions and exclusions. See online supplementary table S1 for detailed baseline characteristics of included and excluded participants.

\section{Baseline characteristics of different groups}

Since measurement of baPWV began with the 20102011 physical examinations, we used data from 2010 to 2011 as the baseline for this study. After the 2-year follow-up, the percentages of groups with a decreased,

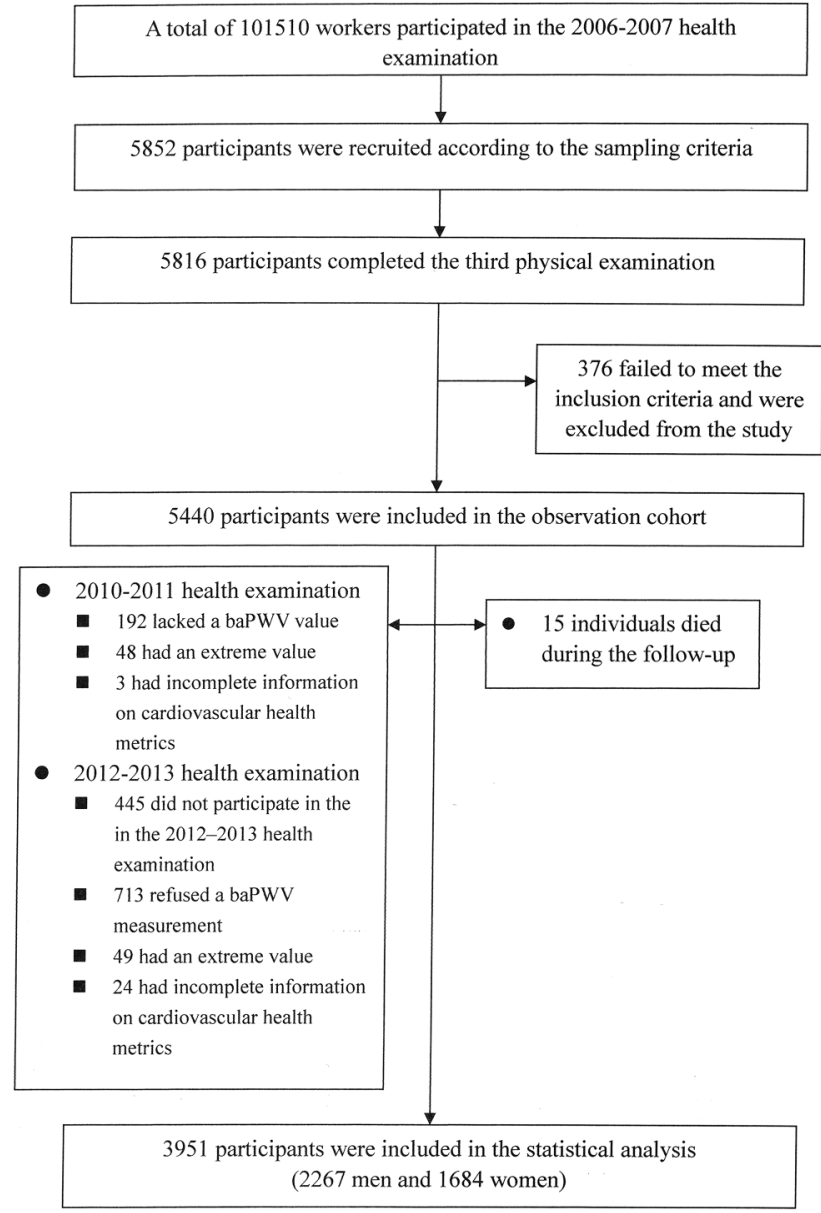

Figure 1 Flow chart of this study (baPWV, brachial-ankle pulse wave velocity).

unchanged and increased CHS values were $44.57 \%$, $22.07 \%$, and $33.36 \%$, respectively. Baseline characteristics of groups with different $\triangle \mathrm{CHS}$ are shown in table 1 . The average age was $53.66 \pm 10.97$ years, and $57.38 \%$ were men. The systolic BP (SBP), diastolic BP (DBP), BMI, TC and FBG were in normal ranges. After 2 years of follow-up, the SBP, TC, FBG and baPWV had increased from baseline; however the DBP, BMI and CHS had decreased from baseline. With $\triangle$ CHS converted from $\leq-4$ to $\geq 3$, all of the items showed an increasing trend ( $p$ for trend $<0.001$ ) while the baseline age reduced gradually. In groups with an increased CHS ( $\triangle \mathrm{CHS}=1, \triangle \mathrm{CHS}=2$ and $\triangle \mathrm{CHS} \geq 3$ ), a more obvious increase of $\triangle \mathrm{CHS}$ resulted in a more significant reduction of $\triangle \mathrm{SBP}, \Delta \mathrm{DBP}, \Delta \mathrm{BMI}, \Delta \mathrm{TC}$ and $\Delta \mathrm{FBG}$.

\section{baPWV of groups with different $\triangle$ CHS}

The baseline baPWV, follow-up baPWV, and $\triangle \mathrm{baPWV}$ of groups with different $\triangle \mathrm{CHS}$ are shown in table 2 . The groups did not differ significantly from one another ( $\mathrm{p}=0.507)$. With $\triangle \mathrm{CHS}$ converted from $\leq-4$ to $\geq 3$ $(\triangle \mathrm{CHS} \leq-4,=-3,=-2,=-1,=0,=1,=2$, and $\geq 3)$, $\triangle \mathrm{baPWV}$ reduced gradually $(126.46 \pm 355.91, \quad 78.4$ $\pm 343.81,69.6 \pm 316.27,49.59 \pm 287.57,57.07 \pm 261.17,40.45$ 
Table 1 Baseline characteristics of groups with different $\triangle \mathrm{CHS}(\mathrm{n}=3951)$

\begin{tabular}{|c|c|c|c|c|c|}
\hline & Total & $\leq-4$ & -3 & -2 & -1 \\
\hline$n$ & 3951 & 171 & 286 & 506 & 798 \\
\hline \multicolumn{6}{|c|}{ Baseline (2010-2011) } \\
\hline Age, year & $53.66 \pm 10.97$ & $57.04 \pm 10.6$ & $55.19 \pm 11.06$ & $53.86 \pm 11.37$ & $54.32 \pm 11.22$ \\
\hline Male (\%) & $2267(57.38)$ & $84(49.12)$ & $140(48.95)$ & $265(52.37)$ & $426(53.38)$ \\
\hline $\mathrm{SBP}, \mathrm{mm} \mathrm{Hg}$ & $129.73 \pm 19.27$ & $124.73 \pm 19.09$ & $126.72 \pm 20.28$ & $127.23 \pm 19.57$ & $128.52 \pm 20.51$ \\
\hline $\mathrm{DBP}, \mathrm{mm} \mathrm{Hg}$ & $82.73 \pm 10.89$ & $77.97 \pm 10.12$ & $80.84 \pm 10.79$ & $80.67 \pm 11.05$ & $81.3 \pm 10.74$ \\
\hline BMI, $\mathrm{kg} / \mathrm{m}^{2}$ & $24.96 \pm 3.22$ & $24.69 \pm 3.02$ & $24.92 \pm 3.05$ & $24.57 \pm 3.22$ & $24.86 \pm 3.18$ \\
\hline $\mathrm{TC}, \mathrm{mg} / \mathrm{dL}$ & $195.77 \pm 38.59$ & $190.09 \pm 37.47$ & $190.9 \pm 34.04$ & $190.47 \pm 34.22$ & $194.72 \pm 36.71$ \\
\hline $\mathrm{FBG}, \mathrm{mg} / \mathrm{dL}$ & $99.88 \pm 25.35$ & $97 \pm 27.7$ & $96.95 \pm 23.69$ & $97.61 \pm 24.98$ & $99.43 \pm 24.11$ \\
\hline $\mathrm{CHS}$ & $8.6 \pm 2.32$ & $10.45 \pm 1.74$ & $10.04 \pm 1.87$ & $9.61 \pm 2.04$ & $9.1 \pm 2.2$ \\
\hline \multicolumn{6}{|c|}{ Changes (2012-2013, 2010-2011) } \\
\hline$\triangle \mathrm{SBP}, \mathrm{mm} \mathrm{Hg}$ & $1.81 \pm 17.44$ & $12.42 \pm 19.63$ & $8.14 \pm 16.29$ & $6.78 \pm 17.05$ & $3.1 \pm 16.7$ \\
\hline$\triangle \mathrm{DBP}, \mathrm{mm} \mathrm{Hg}$ & $-0.46 \pm 12.12$ & $8.24 \pm 14$ & $3.62 \pm 11.37$ & $2.38 \pm 11.84$ & $1.5 \pm 11.54$ \\
\hline$\Delta \mathrm{BMI}, \mathrm{kg} / \mathrm{m}^{2}$ & $-0.04 \pm 2.36$ & $1.6 \pm 4.05$ & $0.57 \pm 2.33$ & $0.31 \pm 2.5$ & $0.16 \pm 2.05$ \\
\hline$\Delta \mathrm{TC}, \mathrm{mg} / \mathrm{dL}$ & $4.33 \pm 52.25$ & $27.81 \pm 32.41$ & $25.36 \pm 117.91$ & $15.73 \pm 31.05$ & $7.41 \pm 33.64$ \\
\hline$\triangle \mathrm{FBG}, \mathrm{mg} / \mathrm{dL}$ & $3.2 \pm 22.1$ & $14.38 \pm 26.13$ & $13.1 \pm 27.77$ & $8.23 \pm 21.23$ & $4.86 \pm 21.29$ \\
\hline \multirow[t]{2}{*}{$\triangle \mathrm{CHS}$} & $-0.28 \pm 1.88$ & $-4.42 \pm 0.77$ & $-3 \pm 0$ & $-2 \pm 0$ & $-1 \pm 0$ \\
\hline & 0 & 1 & 2 & $\geq 3$ & p Value \\
\hline $\mathrm{n}$ & 872 & 681 & 397 & 240 & \\
\hline \multicolumn{6}{|c|}{ Baseline (2010-2011) } \\
\hline Age, years & $53.42 \pm 10.93$ & $52.7 \pm 10.58$ & $52.61 \pm 10.61$ & $52.1 \pm 10.57$ & $<0.001$ \\
\hline Male (\%) & $510(58.49)$ & $412(60.5)$ & $262(65.99)$ & $168(70)$ & $<0.001$ \\
\hline $\mathrm{SBP}, \mathrm{mm} \mathrm{Hg}$ & $130.71 \pm 19.41$ & $131.09 \pm 18.6$ & $132.19 \pm 16.83$ & $134.69 \pm 15.97$ & $<0.001$ \\
\hline $\mathrm{DBP}, \mathrm{mm} \mathrm{Hg}$ & $83.23 \pm 10.88$ & $84.12 \pm 10.7$ & $85.61 \pm 10.05$ & $87.03 \pm 10.28$ & $<0.001$ \\
\hline BMI, $\mathrm{kg} / \mathrm{m}^{2}$ & $24.93 \pm 3.31$ & $25.07 \pm 3.24$ & $25.36 \pm 3.21$ & $25.55 \pm 3.21$ & 0.001 \\
\hline $\mathrm{TC}, \mathrm{mg} / \mathrm{dL}$ & $195.29 \pm 40.31$ & $196.53 \pm 39.1$ & $202.56 \pm 42.12$ & $208.61 \pm 41.2$ & $<0.001$ \\
\hline $\mathrm{FBG}, \mathrm{mg} / \mathrm{dL}$ & $98.03 \pm 21.92$ & $101.65 \pm 26.31$ & $103.58 \pm 27.86$ & $107.34 \pm 31.85$ & $<0.001$ \\
\hline $\mathrm{CHS}$ & $8.44 \pm 2.11$ & $7.98 \pm 2.11$ & $7.25 \pm 2.07$ & $6.27 \pm 2.06$ & $<0.001$ \\
\hline \multicolumn{6}{|c|}{ Changes (2012-2013, 2010-2011) } \\
\hline$\triangle \mathrm{SBP}, \mathrm{mm} \mathrm{Hg}$ & $1.09 \pm 16.6$ & $-1.57 \pm 16.47$ & $-3.14 \pm 16.87$ & $-7.72 \pm 17.04$ & $<0.001$ \\
\hline$\triangle \mathrm{DBP}, \mathrm{mm} \mathrm{Hg}$ & $-0.69 \pm 11.44$ & $-3.34 \pm 10.99$ & $-4.59 \pm 11.44$ & $-8.17 \pm 11.91$ & $<0.001$ \\
\hline$\Delta \mathrm{BMI}, \mathrm{kg} / \mathrm{m}^{2}$ & $-0.18 \pm 1.82$ & $-0.39 \pm 2.01$ & $-0.67 \pm 2.08$ & $-0.86 \pm 3.37$ & $<0.001$ \\
\hline$\Delta \mathrm{TC}, \mathrm{mg} / \mathrm{dL}$ & $0.85 \pm 30.18$ & $-0.97 \pm 66.6$ & $-10.25 \pm 38.6$ & $-20.02 \pm 35.64$ & $<0.001$ \\
\hline$\triangle \mathrm{FBG}, \mathrm{mg} / \mathrm{dL}$ & $3.04 \pm 18.73$ & $-0.5 \pm 17.93$ & $-4.62 \pm 17.82$ & $-8.65 \pm 30.58$ & $<0.001$ \\
\hline$\triangle \mathrm{CHS}$ & $0 \pm 0$ & $1 \pm 0$ & $2 \pm 0$ & $3.5 \pm 0.81$ & $<0.001$ \\
\hline
\end{tabular}

changes ( $\triangle$ ), physical examination metrics of 2012-2013 minus metrics for 2010-2011; baPWV, brachial-ankle pulse wave velocity; BMI, body mass index; CHS, cardiovascular health score; DBP, diastolic blood pressure; FBG, fasting blood glucose; SBP, systolic blood pressure; TC, total cholesterol.

$\pm 264.27,37.45 \pm 283.26$ and $21.66 \pm 264.17 \mathrm{~cm} / \mathrm{s}$, respectively; $\mathrm{p}$ for trend $<0.05)$. A more obvious increase in $\triangle$ CHS was associated with a steeper reduction in $\triangle \mathrm{baPWV}$ (see table 1 for details). In addition, when $\triangle \mathrm{CHS} \geq 4$, the baPWV did not increase further but rather decreased gradually $(-29.90 \pm 215.92 \mathrm{~cm} / \mathrm{s}$ ) (see online supplementary table S2 for details). Although no statistical significance was achieved, we still detected a trend of an inverse relationship between $\triangle \mathrm{CHS}$ and $\triangle \mathrm{baPWV}$.

\section{Linear regression analysis between $\triangle$ CHS and $\triangle$ baPWV}

The linear regression analysis was performed with $\triangle \mathrm{baPWV}$ as a dependent variable and $\triangle \mathrm{CHS}$ as the independent variable. Mode 1: This was a single-factor analysis model. Mode 2: On the basis of Mode 1, we further adjusted for age and gender. Mode 3: On the basis of Mode 2, we further adjusted for baseline CHS and baseline baPWV. The results of mode 3 suggested that age were positively associated with $\triangle \mathrm{baPWV}$. Female gender, baseline CHS, baseline baPWV, and $\triangle \mathrm{CHS}$ were negatively associated with $\triangle \mathrm{baPWV}$. With adjustments for age, gender, baseline CHS, and baseline baPWV, $\triangle \mathrm{baPWV}$ decreased with increasing $\triangle \mathrm{CHS}$. Each increase in $\triangle \mathrm{CHS}$ was associated with a $15.22 \mathrm{~cm} / \mathrm{s}$ decrease in $\triangle \mathrm{baPWV}$ (B value $-15.22, \mathrm{p}<0.001$; table 3 ). The relationships between each individual cardiovascular health component and $\triangle \mathrm{baPWV}$ were also analysed by using linear regression model (see online supplementary table S3 for details).

\section{Sensitivity analysis}

To eliminate the influence of missing value replacement, we excluded participants with missing values and 
Table 2 baPWV of groups with different $\triangle \mathrm{CHS}$

\begin{tabular}{llllll}
\hline & Total & $\leq-4$ & $\mathbf{- 3}$ & $\mathbf{- 2}$ & \multicolumn{1}{c}{$\mathbf{1}$} \\
\hline $\mathrm{N}$ & 3951 & 171 & 286 & 506 & 798 \\
Baseline baPWV, cm/s & $1535.04 \pm 336.88$ & $1562.47 \pm 314.23$ & $1567.21 \pm 368.95$ & $1538.97 \pm 336.57$ & $1535.63 \pm 352.98$ \\
Follow-up baPWV, cm/s & $1589.77 \pm 403.25$ & $1688.92 \pm 436.74$ & $1645.61 \pm 458.72$ & $1608.58 \pm 436.8$ & $1585.21 \pm 404.86$ \\
$\begin{array}{l}\Delta \text { baPWV, cm/s } \\
\text { baPWV groups }\end{array}$ & $54.72 \pm 288.47$ & $126.46 \pm 355.91$ & $78.4 \pm 343.81$ & $69.6 \pm 316.27$ & $49.59 \pm 287.57$ \\
$\begin{array}{l}\text { Non-increasing group, } \mathrm{n}(\%) \\
\text { Increasing group, } \mathrm{n}(\%)\end{array}$ & $1659(41.99)$ & $67(39.18)$ & $117(40.91)$ & $206(40.71)$ & $341(42.73)$ \\
& $2292(58.01)$ & $104(60.82)$ & $169(59.09)$ & $300(59.29)$ & $457(57.27)$
\end{tabular}

\begin{tabular}{|c|c|c|c|c|c|}
\hline & 0 & 1 & 2 & $\geq 3$ & p Value \\
\hline $\mathrm{n}$ & 872 & 681 & 397 & 240 & \\
\hline Baseline baPWV, cm/s & $1529.53 \pm 335.94$ & $1528.66 \pm 336.06$ & $1533.7 \pm 322.67$ & $1507.29 \pm 283.35$ & 0.507 \\
\hline Follow-up baPWV, cm/s & $1586.6 \pm 395.12$ & $1569.11 \pm 385.76$ & $1571.14 \pm 369.94$ & $1528.95 \pm 339.08$ & 0.001 \\
\hline$\triangle \mathrm{baPWV}, \mathrm{cm} / \mathrm{s}$ & $57.07 \pm 261.17$ & $40.45 \pm 264.27$ & $37.45 \pm 283.26$ & $21.66 \pm 264.17$ & 0.005 \\
\hline$\triangle \mathrm{baPWV}$ groups & & & & & 0.518 \\
\hline Decreasing group, $\mathrm{n}(\%)$ & 364 (41.74) & $286(42)$ & 168 (42.32) & $110(45.83)$ & \\
\hline Increasing group, $\mathrm{n}(\%)$ & $508(58.26)$ & $395(58)$ & $229(57.68)$ & $130(54.17)$ & \\
\hline
\end{tabular}

$\triangle$ baPWV, baPWV of the physical examination in 2012-2013 minus baPWV of 2010-2011; baseline baPWV, baPWV of physical examination in 2010-2011; follow-up baPWV, baPWV of the physical examination in 2012-2013; increasing group, $\triangle$ baPWV >0; non-increasing group, $\triangle \mathrm{baPWV} \leq 0$. baPWV, brachial-ankle pulse wave velocity; CHS, cardiovascular health score; $\triangle \mathrm{CHS}, \mathrm{CHS}$ of the physical examination in 2012-2013 minus CHS in 2010-2011.

performed a statistical analysis of data without replacement. The results remain unchanged. The new analysis showed a result similar to that of the original analysis and indicated an obvious negative relationship between $\triangle \mathrm{CHS}$ and $\triangle \mathrm{baPWV}$. With adjustments for age, gender, baseline CHS and baseline baPWV, each increase in $\triangle \mathrm{CHS}$ was associated with an $11.32 \mathrm{~cm} / \mathrm{s}$ decrease in $\triangle$ baPWV $(\mathrm{p}<0.001$; table 4$)$.

\section{DISCUSSION}

Since the AHA proposed seven metrics of cardiovascular health behaviours and health factors, several studies have suggested that ideal cardiovascular health metrics plays an important role in protection of the cardiovascular and cerebrovascular systems. ${ }^{3}{ }^{6}$ With an increasing value of cardiovascular health metrics, highsensitivity $\mathrm{C}$ reactive protein, ${ }^{17}$ carotid artery intimamedia thickness ${ }^{18} 19$ and the incidence of cardiovascular and cerebrovascular events ${ }^{36}$ decrease gradually. In addition, improvement in cardiovascular health status can reduce the risk of subclinical atherosclerosis and cardiovascular events. ${ }^{720}$ Arterial stiffness is a predictor of atherosclerosis and CVD, ${ }^{9}$ which can be measured by PWV. ${ }^{10}$ Carotid-femoral pulse wave velocity (cfPWV) is the gold standard for measuring arterial stiffness and is reported to predict future mortality ${ }^{21}$ and morbidity ${ }^{9}$ from CVD, but its measurement is fairly complicated and time-consuming. However, baPWV measures have an excellent correlation with cfPWV and can reflect the

Table 3 Linear regression analysis between $\triangle \mathrm{CHS}$ and $\triangle$ baPWV

\begin{tabular}{|c|c|c|c|c|}
\hline & $B$ value & $95 \% \mathrm{Cl}$ & $\boldsymbol{\beta}$ & p Value \\
\hline \multicolumn{5}{|l|}{ Mode 1} \\
\hline$\Delta \mathrm{CHS}$ & -10.06 & -14.84 to -5.27 & -0.07 & 0.000 \\
\hline \multicolumn{5}{|l|}{ Mode 2} \\
\hline$\Delta \mathrm{CHS}$ & -10.56 & -15.41 to -5.71 & -0.07 & 0.000 \\
\hline Baseline age & 0.62 & -0.22 to 1.46 & 0.02 & 0.150 \\
\hline Female & -27.37 & -46.03 to -8.72 & -0.05 & 0.000 \\
\hline \multicolumn{5}{|l|}{ Mode 3} \\
\hline$\Delta \mathrm{CHS}$ & -15.22 & -20.43 to -10.02 & -0.10 & 0.000 \\
\hline Baseline age & 6.76 & 5.71 to 7.81 & 0.26 & 0.000 \\
\hline Female & -28.28 & -47.73 to -8.84 & -0.05 & 0.004 \\
\hline Baseline CHS & -10.98 & -15.68 to -6.29 & -0.09 & 0.000 \\
\hline Baseline baPWV & -0.32 & -0.36 to -0.29 & -0.38 & 0.000 \\
\hline \multicolumn{5}{|c|}{$\begin{array}{l}\triangle \text { baPWV, baPWV of the fourth physical examination minus baPWV of the third physical examination; } \triangle \mathrm{CHS} \text {, CHS of the fourth physical } \\
\text { examination minus CHS of the third physical examination; mode } 1 \text {, a single-factor analysis model; mode } 2 \text {, adjusted for age and gender on } \\
\text { the basis of mode } 1 \text {; mode } 3 \text {, adjusted for baseline CHS and baseline baPWV on the basis of mode } 2 \text {. } \\
\text { baPWV, brachial-ankle pulse wave velocity; CHS, cardiovascular health score. }\end{array}$} \\
\hline
\end{tabular}


Table 4 Sensitivity analysis

\begin{tabular}{|c|c|c|c|c|}
\hline & B value & $95 \% \mathrm{Cl}$ & $\boldsymbol{\beta}$ & p Value \\
\hline \multicolumn{5}{|l|}{ Mode 3} \\
\hline$\triangle \mathrm{CHS}$ & -11.32 & -17.25 to -5.40 & -0.08 & 0.000 \\
\hline Baseline age & 6.83 & 5.48 to 8.18 & 0.23 & 0.000 \\
\hline Female & -43.63 & -66.40 to -20.87 & -0.08 & 0.004 \\
\hline Baseline CHS & -9.15 & -14.82 to -3.48 & -0.08 & 0.002 \\
\hline Baseline baPWV & -0.31 & -0.35 to -0.27 & -0.34 & 0.000 \\
\hline
\end{tabular}

$\triangle \mathrm{baPWV}$, baPWV of the fourth physical examination minus baPWV of the third physical examination; $\triangle \mathrm{CHS}, \mathrm{CHS}$ of the fourth physical examination minus $\mathrm{CHS}$ of the third physical examination; mode, adjusted for multiple factors.

baPWV, brachial-ankle pulse wave velocity; CHS, cardiovascular health score.

elasticity of the large and the medium arterial systems; furthermore, baPWV measurement is much more convenient and requires only a short period of time. ${ }^{11}$ The relationships between baPWV and CVD mortality in the older population, total mortality in the general population, ${ }^{12}$ CVD incidence ${ }^{14}$ and CVD risk $^{15}$ have been reported in several studies. It is most likely that the protective effect of cardiovascular health status improvement on the incidence of CVD might be mediated by its favourable effects on baPWV.

The influencing factors of baPWV are rather complicated. Numbers of metabolic syndrome components, ${ }^{22}$ body size phenotypes, ${ }^{23}$ ageing and male gender, ${ }^{24}$ aerobic exercise and smoking ${ }^{25}$ as well as $\mathrm{BP}$ control $^{26}$ were reported to impact on baPWV. Therefore, evaluating the effect of each factor is difficult to achieve. For this reason, a comprehensive estimate system is needed to investigate the relationship between baPWV and multiple influencing factors. The AHA CHS, based on four health behaviours and three health factors, covers a variety of influences. Thus, analysing the relationship between $\triangle \mathrm{CHS}$ and $\triangle \mathrm{baPWV}$ might be a useful tool, but the relationship between them has rarely been reported. As far as we know, our study is the first large-scale investigation aimed at exploring this association. The outcomes of our study indicated that $\triangle$ baPWV decreased gradually with increasing $\triangle \mathrm{CHS}$. In addition, a more obvious CHS decrease was associated with a more significant baPWV increase, and conversely, a more obvious CHS increase resulted in attenuation of the baPWV increase. Indeed, at $\triangle \mathrm{CHS} \geq 4$, baPWV stopped increasing and began a gradual decrease. These results suggest that an improved CHS can reduce the progression of atherosclerosis, as measured by baPWV. The negative relationship between $\triangle \mathrm{CHS}$ and $\triangle \mathrm{baPWV}$ remains unchanged after adjusting for age, gender, baseline CHS and baseline baPWV. Each increase in $\triangle \mathrm{CHS}$ was associated with a $\triangle \mathrm{baPWV}$ decrease of $15.22 \mathrm{~cm} / \mathrm{s}$. Previous studies have shown that atherosclerosis is a reversible process. Diet, regular exercise and lipid-lowering treatment might reverse the progression of atherosclerosis. ${ }^{27}$ Our present findings confirmed this as well: individuals with increased CHS experienced a reduced atherosclerosis progression. Moreover, the progression was reversed among people with $\Delta \mathrm{CHS} \geq 4$.
Previous work from Aatola $e t a l^{29}$ estimated changes in ideal cardiovascular health status among children and youth. The results showed that a change in ideal cardiovascular health status, from childhood or young adulthood, was an independent predictor of adult PWV; however, whether the beneficial effect could also be found in middle-aged and older people was unknown. The current results suggest that an improvement in cardiovascular health status plays an important role for middle-aged and older people, with a favourable effect that appears in a short period of time. Low prevalence and poor improvement of ideal cardiovascular health was reported in several studies. Only $5 \%$ of the population meets the criteria for ideal cardiovascular health in the USA. ${ }^{2}$ The percentage of individuals with an increased CHS in the CARDIA study was 25.3\%.7 A similar trend was also found in China by $\mathrm{Wu}$ et $a .^{8}$ Only $0.6 \%$ of male participants and $2.6 \%$ of female participants ( $\mathrm{n}=1012$ 418) met the 'ideal' for all seven health components. The reason for a higher improvement ratio $(33.36 \%)$ in our study might be the regular health education and physical examination among workers in the Kailuan Group Corporation. If this practice and experience were generalised to the entire Chinese population, a more significant benefit to the cardiovascular and cerebrovascular system might be achieved.

This study has some limitations. First, we did not completely adhere to the health indicators proposed by AHA. Since there were no specific data about diet, we adopted salt intake as a proxy, which could have led to an underestimate of the influence of diet on $\triangle \mathrm{baPWV}$; however, evidence has shown that salt intake among Chinese people is much higher than in other countries. ${ }^{30}$ Thus, the amount of salt intake is likely to be of greater significance than other indicators of diet in the Chinese population. Second, the duration of follow-up was not long enough to fully track the progression of atherosclerosis. Nevertheless, a favourable effect of an improved CHS emerged even during the relatively short follow-up. Third, the results of our study are based on middle-aged and older people, but whether it can be generalised to the whole population warrants further validation.

In this prospective cohort study, we offer the first evidence of an inverse relationship between CHS 
improvement and baPWV. Each increase in CHS was associated with a reduced baPWV of $15.22 \mathrm{~cm} / \mathrm{s}$, which proved to be an independent predictor of atherosclerosis progression. These findings might partly explain the negative association between CHS and CVD incidence. Therefore, improving CHS and maintaining a healthy lifestyle can attenuate or even reverse the progression of atherosclerosis, which might partly explain the mechanism of an inverse relationship between CHS and incidence of CVD.

Contributors JG, MB, YL, JS, ZH, AX, YW, SA, XY, SW and JC participated in the study design, drafting of the manuscript, data collection and statistical analysis. SW and XY gave final approval for the manuscript.

Funding This work is supported by grants from the National Basic Research Program of China (973 program), (no. 2014CB542302); National Natural Science Foundation of China (no. 811170244, 81222001, 81271841, 81470541); and Scientific Research Common Program of Beijing Municipal Commission of Education (no. KM 201310025028).

Competing interests None declared.

Patient consent Obtained.

Ethics approval The Ethics Committee of the Kailuan General Hospital and Beijing Chaoyang Hospital.

Provenance and peer review Not commissioned; externally peer reviewed.

Data sharing statement Additional data can be accessed via the Dryad data repository at http://datadryad.org/with the doi: 10.5061/dryad.8h6f2/1.

Open Access This is an Open Access article distributed in accordance with the terms of the Creative Commons Attribution (CC BY 4.0) license, which permits others to distribute, remix, adapt and build upon this work, for commercial use, provided the original work is properly cited. See: http:// creativecommons.org/licenses/by/4.0/

\section{REFERENCES}

1. Xu J, Kochanek KD, Murphy SL, et al. Deaths: final data for 2007. National Vital Statistics Reports. 2010;58:19.

2. Lloyd-Jones DM, Hong Y, Labarthe D, et al. American Heart Association Strategic Planning Task F and Statistics C. Defining and setting national goals for cardiovascular health promotion and disease reduction: the American Heart Association's strategic Impact Goal through 2020 and beyond. Circulation 2010;121:586-613.

3. Folsom AR, Yatsuya H, Nettleton JA, et al. ARIC Study Investigators. Community prevalence of ideal cardiovascular health, by the American Heart Association definition, and relationship with cardiovascular disease incidence. J Am Coll Cardiol 2011;57:1690-6.

4. van Dam RM, Li T, Spiegelman D, et al. Combined impact of lifestyle factors on mortality: prospective cohort study in US women. BMJ 2008;337:a1440.

5. Huffman MD, Capewell S, Ning $\mathrm{H}$, et al. Cardiovascular health behavior and health factor changes (1988-2008) and projections to 2020: results from the National Health and Nutrition Examination Surveys. Circulation 2012;125:2595-602.

6. Dong C, Rundek T, Wright CB, et al. Ideal cardiovascular health predicts lower risks of myocardial infarction, stroke, and vascular death across whites, blacks, and Hispanics: the northern Manhattan study. Circulation 2012;125:2975-84.

7. Spring B, Moller AC, Colangelo LA, et al. Healthy lifestyle change and subclinical atherosclerosis in young adults: Coronary Artery Risk Development in Young Adults (CARDIA) study. Circulation 2014;130:10-17.

8. Wu HY, Sun ZH, Cao DP, et al. Cardiovascular health status in Chinese adults in urban areas: analysis of the Chinese Health Examination Database 2010. Int J Cardiol 2013;168:760-4.
9. Vlachopoulos C, Aznaouridis K, Stefanadis C. Prediction of cardiovascular events and all-cause mortality with arterial stiffness: a systematic review and meta-analysis. J Am Coll Cardiol 2010;55:1318-27.

10. Lehmann ED. Clinical value of aortic pulse-wave velocity measurement. Lancet 1999;354:528-9.

11. Cortez-Cooper MY, Supak JA, Tanaka H. A new device for automatic measurements of arterial stiffness and ankle-brachial index. Am J Cardiol 2003;91:1519-22.

12. Miyano I, Nishinaga M, Takata J, et al. Association between brachial-ankle pulse wave velocity and 3-year mortality in community-dwelling older adults. Hypertens Res 2010;33:678-82.

13. Turin TC, Kita $Y$, Rumana N, et al. Brachial-ankle pulse wave velocity predicts all-cause mortality in the general population: findings from the Takashima study, Japan. Hypertens Res 2010;33:922-5.

14. Takashima N, Turin TC, Matsui K, et al. The relationship of brachial-ankle pulse wave velocity to future cardiovascular disease events in the general Japanese population: the Takashima study. J Hum Hypertens 2014;28:323-7.

15. Vlachopoulos C, Aznaouridis K, Terentes-Printzios D, et al. Prediction of cardiovascular events and all-cause mortality with brachial-ankle elasticity index: a systematic review and meta-analysis. Hypertension 2012;60:556-62.

16. Wu S, Huang $Z$, Yang $X$, et al. Prevalence of ideal cardiovascular health and its relationship with the 4-year cardiovascular events in a northern Chinese industrial city. Circ Cardiovasc Qual Outcomes 2012;5:487-93.

17. Kim JI, Sillah A, Boucher JL, et al. Prevalence of the American Heart Association's "Ideal Cardiovascular Health" Metrics in a Rural, Cross-sectional, Community-Based Study: The Heart of New UIm Project. J Am Heart Assoc 2013;2:e000058.

18. Oikonen M, Laitinen TT, Magnussen CG, et al. Ideal cardiovascular health in young adult populations from the United States, Finland, and Australia and its association with cIMT: the International Childhood Cardiovascular Cohort Consortium. J Am Heart Assoc 2013;2:e000244.

19. Kulshreshtha A, Goyal A, Veledar E, et al. Association between ideal cardiovascular health and carotid intima-media thickness: a twin study. J Am Heart Assoc 2014;3:e000282.

20. Xanthakis V, Enserro DM, Murabito JM, et al. Ideal cardiovascular health: associations with biomarkers and subclinical disease, and impact on incidence of cardiovascular disease in the Framingham Offspring Study. Circulation 2014;130:1676-83.

21. Inoue N, Maeda R, Kawakami H, et al. Aortic pulse wave velocity predicts cardiovascular mortality in middle-aged and elderly Japanese men. Circ J 2009;73:549-53.

22. Zhou F, Zhang H, Yao W, et al. Relationship between brachial-ankle pulse wave velocity and metabolic syndrome components in a Chinese population. J Biomed Res 2014;28:262-8.

23. Yoo HJ, Hwang SY, Hong HC, et al. Association of metabolically abnormal but normal weight (MANW) and metabolically healthy but obese (MHO) individuals with arterial stiffness and carotid atherosclerosis. Atherosclerosis 2014;234:218-23.

24. Uemura $\mathrm{H}$, Katsuura-Kamano $\mathrm{S}$, Yamaguchi $\mathrm{M}$, et al. Family history of stroke is potentially associated with arterial stiffness in the Japanese population. Arch Cardiovasc Dis 2014:107:654-63.

25. Park W, Miyachi M, Tanaka H. Does aerobic exercise mitigate the effects of cigarette smoking on arterial stiffness? J Clin Hypertens (Greenwich) 2014:16:640-4.

26. Chung CM, Cheng HW, Chang JJ, et al. Relationship between resistant hypertension and arterial stiffness assessed by brachial-ankle pulse wave velocity in the older patient. Clin Inter Aging 2014;9:1495-502.

27. Weisbrod RM, Shiang T, Al Sayah L, et al. Arterial stiffening precedes systolic hypertension in diet-induced obesity. Hypertension 2013;62:1105-10

28. Ahmed $\mathrm{HM}$, Blaha MJ, Nasir $\mathrm{K}$, et al. Effects of physical activity on cardiovascular disease. Am J Cardiol 2012;109:288-95.

29. Aatola $\mathrm{H}$, Hutri-Kahonen $\mathrm{N}$, Juonala $\mathrm{M}$, et al. Prospective relationship of change in ideal cardiovascular health status and arterial stiffness: the Cardiovascular Risk in Young Finns Study. J Am Heart Assoc 2014;3:e000532.

30. Yan L, Xu J, Zhang M, et al. Analysis of self-reported daily salt intake per capital of resident households in China, 2010. 2014. 